\title{
Pityriasis amiantacea: a study of seven cases*
}

Gustavo Moreira Amorim ${ }^{1}$

\author{
Nurimar Conceição Fernandes ${ }^{1}$
}

DOI: http://dx.doi.org/10.1590/abd1806-4841.20164951

\begin{abstract}
Pityriasis amiantacea was first described in 1832. The disease may be secondary to any skin condition that primarily affects the scalp, including seborrheic dermatitis. Its pathogenesis remains uncertain. We aim to analyze the epidemiological and clinical profiles of patients with pityriasis amiantacea to better understand treatment responses. We identified seven cases of pityriasis amiantacea and a female predominance in a sample of 63 pediatric patients with seborrheic dermatitis followed for an average of 20.4 months. We reported a mean age of 5.9 years. Five patients were female, with a mean age of 9 years. All patients were successfully treated with topic ketoconazole.
\end{abstract}

Keywords: Dermatitis, seborrheic; Pityriasis; Tinea

Pityriasis amiantacea (PA) can be described as an exaggerated inflammatory response pattern that affects the scalp, secondary to any dermatitis that may affect that region. It was first described by Alibert in 1832 as a condition characterized by thick silvery or yellowish scales, resembling asbestos fibers, strongly adhered to tufts of hair. ${ }^{1}$ Frequency data for the disease are scarce in the literature and its etiopathogenesis remains unclear. Patients possibly present some sort of genetic or environmental predisposition for the disease. ${ }^{1,2}$ Among the possible causes, we underline atopic and seborrheic dermatitis, psoriasis, and tinea capitis. However, it is known that the disease presents no associated dermatitis in certain cases. ${ }^{3,4}$

The aim of this study is to analyze the epidemiological and clinical profiles of patients with pityriasis amiantacea to better understand treatment responses.

This is a cross-sectional and longitudinal study performed with children and adolescents affected by seborrheic dermatitis and pityriasis amiantacea treated at the dermatology pediatric outpatient clinic of the Instituto de Pediatria e Puericultura Matergão Gesteira (IPPMG) from January 2004 to December 2011. Data were obtained through a review of medical records. Inclusion criteria for seborrheic dermatitis were: erythema and scaling of the scalp, with or without advancement to the retroauricular regions; presence or absence of erythematous scaly lesions in other seborrheic areas; and absence of atopic eczematous lesions or psoriasis. Inclusion criteria for pityriasis amiantacea were silvery or yellowish scales encircling hair shafts, direct mycological examination and positive or negative culture from scale or hair samples.

Sixty-three patients with seborrheic dermatitis were treated during the period, with a median follow-up time of 20.4 months (SD: 23.6 months). We noticed a female predominance in the sample $(38 / 63 ; 60.3 \%)$ at mean age of 5.9 years (SD: 4.2 years). All patients presented with scalp involvement: in 26 (41.2\%) eczema was restricted to this area and in 37 (58.7\%) erythematous scaly lesions spread onto other seborrheic areas (central face, ear, anterior chest wall, and inguinogenital region). During follow-up, seven patients with seborrheic dermatitis revealed pityriasis amiantacea as a complication (11.1\% or $7 / 63$ ) (Table 1$)$. Among these patients, five were female $(5 / 7,71.4 \%)$ with a mean age at diagnosis of nine years (SD:

Received on 20.07.2015

Approved by the Advisory Board and accepted for publication on 28.10.2015

* Work performed at Instituto de Puericultura e Pediatria Matergão Gesteira - Universidade Federal do Rio de Janeiro (IPPMG-UFRJ) - Rio de Janeiro (RJ), Brazil.

Financial Support: None.

Conflict of Interest: None.

1 Universidade Federal do Rio de Janeiro (UFRJ) - Rio de Janeiro (RJ), Brazil.

(C2016 by Anais Brasileiros de Dermatologia 
TABLE 1: Characteristics of patients with seborrheic eczema cared for during the period

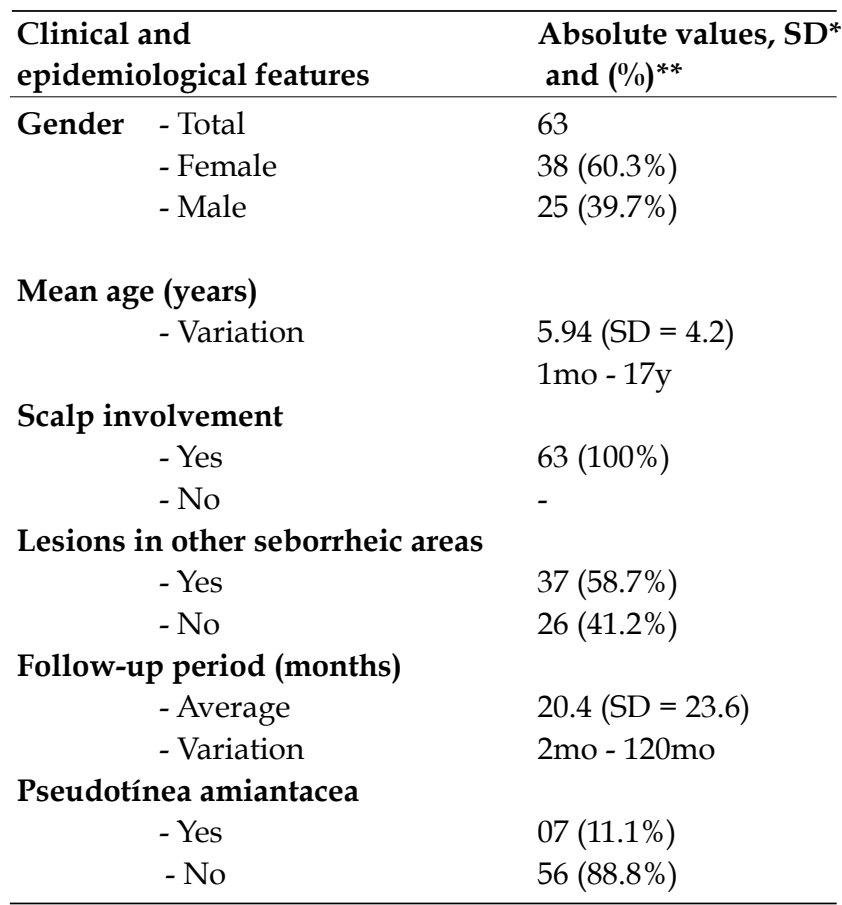

* Standard deviation (SD).

2.8 years). Clinically, all cases were associated with pruritus and erythema. Only one case showed scarring alopecia $(1 / 7,14.2 \%)$. Direct mycological examination and culture of scales and hair were negative. We opted for ketoconazole $2 \%$ shampoo, which is associated with topical mineral oil for removal of scales, as treatment for all pityriasis amiantacea patients. The average treatment time with daily application was 6.4 months $(\mathrm{SD}=2.6$ months). We also administered topical high-potency steroids (betamethasone dipropionate $0.1 \%$ solution) to 2 patients $(28.4 \%)$. All seven patients responded fully to the treatment, with a mean follow-up of 9.8 months (SD = 8.2 months) (Table 2).

Some authors suggest that pityriasis amiantacea is a possible complication in infants with seborrheic dermatitis. ${ }^{5}$ Others are emphatic in pointing out that the reaction is more common among children although it can occur at any age. ${ }^{6}$ The prospective study of 85 PA cases revealed a mean age diagnosis of 23.8 years in the interval ranging from 5 to 63 - thus encompassing children and adolescents, with a female predominance. In the present study, psoriasis accounted for $35.3 \%$ of the cases; aggregated seborrheic dermatitis and atopic eczema for $34.2 \%$; tinea capitis - diagnosed by mycological examination - for $12.9 \%$ of cases. ${ }^{2}$ Our research revealed that $11.1 \%$ of seborrheic dermatitis patients had pityriasis amiantacea during follow-up. Unlike the $42 \%$ described in the literature, ${ }^{5}$ all 63 patients presented with scalp involvement in our study. In relation to diagnostic investigation, a scalp biopsy was probably not performed because histopathology is not presented as a fundamental
TABLE 2: Sample profile of patients with pityriasis amiantacea

\begin{tabular}{lc}
\hline $\begin{array}{l}\text { Clinical and } \\
\text { epidemiological features }\end{array}$ & $\begin{array}{c}\text { Absolute values, } \text { SD }^{*} \\
\text { and }(\mathbf{( \% )})^{* *}\end{array}$ \\
\hline Gender - Total & 07 \\
- Female & $05(71.4 \%)$ \\
- Male & $02(28.6 \%)$ \\
Mean age (years) & \\
- Variation & $9(\mathrm{SD}=2.8)$ \\
Symptom duration (months) & $5 \mathrm{y}-14 \mathrm{y}$ \\
- Average & $12(\mathrm{SD}=21.2)$ \\
- Variation & $1 \mathrm{mo}-60 \mathrm{mo}$
\end{tabular}

Involvement Topography - localized

- Diffuse

$07(100 \%)$

Associated Erythema

- Yes

$07(100 \%)$

- No

$-$

Associated itching

- Yes

$07(100 \%)$

- No

$06(85.8 \%)$

Alopecia - Non-cicatrical

$01(14.2 \%)$

- Cicatrical

Mycological examination (scale and hair shaft)

- Negative

$07(100 \%)$

- Positive

Fungal culture (scale and hair shaft)

- Negative

$-$

- Positive

$07(100 \%)$

Diagnosis - seborrheic eczema

$07(100 \%)$

$$
\text { - Others }
$$

\section{Treatment}

- $2 \%$ ketoconazole shampoo

$07(100 \%)$

- Topic associated corticosteroids

$02(28.6 \%)$

Treatment time (months)

- Average

$6.4(\mathrm{SD}=2.6)$

- Variation

$4-11$

Response - Full

$07(100 \%)$

- Partial

Follow-up after treatment (months)
- Average
$9.8(\mathrm{SD}=8.2)$
- Variation
$2 \mathrm{mo}-24 \mathrm{mo}$ 
diagnostic criterion for the disease. ${ }^{4}$ All the patients responded adequately to isolated topical treatment. The literature suggests systemic corticosteroids as a therapeutic possibility for non-responders. In cases of documented coinfection, systemic antibiotic with staphylococcus-specific spectrum could be the indicated treatment option. ${ }^{4}$

\section{REFERENCES}

1. Verardino GC, Azulay-Abulafia L, Macedo PM, Jeunon T. Pityriasis amiantacea: clinical-dermatoscopic features and microscopy of hair tufts. An Bras Dermatol. 2012;87:142-5

2. Abdel-Hamid IA, Agha SA, Moustafa YM, EI-Labban AM. Pityriasis amiantacea: a clinical and etiopathologic study of 85 patients. Int J Dermatol. 2003;42:260-4.

3. Ginarte M, Pereiro M Jr, Fernández-Redondo V, Toribio J. Case reports. Pityriasis amiantacea as manifestation of tinea capitis due to microsporum canis. Mycoses. 2000;43:93-6.

4. Fitzpatrick TB, Wolff K, Goldsmith LA, Katz Sl, Gilcherest BA, Paller AS, et al Dermatology in general medicine. 7.ed. New York: Mcgraw-Hill Medical; 2008.

5. Sampaio ALB, Mameri A, Jeunon T, Ramos-e-Silva M, Nunes AP, Carneiro S. Dermatite seborreica. An Bras Dermatol. 2011;86:1061-74.

6. Pereira JM. Tricologia - Avaliação dos pelos, doenças congênitas e hereditárias. In: Belda Junior W, Di Chiacchio N, Criado PR, editores. Tratado de Dermatologia. 2. ed. São Paulo: Editora Atheneu; 2014. p. 945-90.
Since it was first described in 1832, little new information has been published about the epidemiology and pathophysiology of pityriasis amiantacea. From this series of cases involving children and adolescents, we seek to draw attention to the recognition of this condition.]
MAILING ADDRESS:

Gustavo Moreira Amorim

Av. Nossa Senhora de Copacabana, 1418 - Apto 402

Copacabana

22070-012 - Rio de Janeiro - RJ

Brazil

E-mail: gustavomoreiraamorim@hotmail.com

How to cite this article: Amorim GM, Fernandes NC. Pityriasis amiantacea: a study of seven cases. An Bras Dermatol. 2016;91(5):694-6. 